\begin{tabular}{lc|c|c|c|}
\hline Bentham OPEN & The Open Rheumatology Journal \\
\hline CrossMark & Content list available at: www.benthamopen.com/TORJ/ & $\begin{array}{l}\text { The Open } \\
\text { Rheumatology } \\
\text { lournal }\end{array}$ \\
\hline
\end{tabular}

REVIEW ARTICLE

\title{
A Review of Chikungunya Virus-induced Arthralgia: Clinical Manifestations, Therapeutics, and Pathogenesis
}

\author{
$\operatorname{Brad}_{\text {A. Goupil }}{ }^{1}$ and Christopher N. Mores ${ }^{1,2, *}$ \\ ${ }^{I}$ Department of Pathobiological Sciences, Louisiana State University School of Veterinary Medicine, Skip Bertman \\ Drive, Baton Rouge, Louisiana, United States of America \\ ${ }^{2}$ Virology and Emerging Infections, US Naval Medical Research Unit No. 6, Lima Pampa, Peru
}

Received: May 04, 2016

Revised: November 08, 2016

Accepted: November 09, 2016

\begin{abstract}
:
Background:

Chikungunya virus (CHIKV) is a mosquito-borne alphavirus that circulates predominantly in tropical and subtropical regions, potentially affecting over 1 billion people. Recently, an outbreak began in the western hemisphere and has resulted in over 1.8 million reported suspected cases. Infection often results in severe fever, rash and debilitating polyarthralgia lasting weeks to months. Additionally, the current literature reports that CHIKV can result in a severe chronic arthralgia and/or arthritis that can last months to years following the initial infection.
\end{abstract}

\section{Objective:}

The purpose of this review is to evaluate the literature and summarize the current state of knowledge regarding CHIKV-associated disease, including clinical presentation, diagnosis, risk factors for development of severe disease, treatment, and pathogenesis in human patients. Additionally, recommendations are presented regarding avenues for clinical research to help further elucidate the pathogenesis of joint disease associated with CHIKV infection.

\section{Conclusion:}

While there is an association between initial CHIKV infection and acute disease, a causal relationship with development of chronic arthralgia has not been established at this time. Potential causes of chronic CHIKV-induced arthritis have been postulated, including viral persistence, induction of autoimmune disease, and exacerbation of pre-existing joint disease. While there are numerous reports of chronic CHIKV-associated arthralgia and/or arthritis, there is currently no evidence of a definitive link between initial infection and development of chronic disease. Additional, prospective clinical research on CHIKV-associated disease is necessary to further determine the potential role of virus and development of chronic joint disease.

Keywords: Acute, Advanced imaging, Arthralgia, Arthritis, Chikungunya virus, Chronic, Pathogenesis, Therapeutics.

\section{INTRODUCTION}

\section{Background}

Chikungunya virus (CHIKV) is an arbovirus spread predominantly by Aedes aegypti and Ae. albopictus mosquitoes [1, 2]. It belongs to the alphavirus genus along with other arthritogenic viruses such as Ross River, Sindbis, O'nyongnyong, Mayaro, Semliki forest, and Barmah forest viruses [1, 3, 4]. CHIKV is considered a neglected tropical disease, because it circulates within these subtropical and tropical regions, has the potential to affect more than 1 billion people,

\footnotetext{
* Address correspondence to this author at the Department of Pathobiological Sciences, Louisiana State University School of Veterinary Medicine, Skip Bertman Drive, Baton Rouge, Louisiana, United States of America, 70803; Tel: 225-578-9936; Email: cmores@1su.edu
} 
and many at-risk people live in poverty stricken regions [5]. Recently, CHIKV-induced disease was named a nationally notifiable condition in the United States, because of a severe ongoing outbreak in the western hemisphere [6]. The outbreak initially began in October of 2013 and has since spread throughout the western hemisphere, affecting 45 countries and territories and causing approximately 1.8 million reported suspected cases as of March 2016 [7]. In 2014, 2,811 cases of CHIKV-induced disease were reported in the United States, which included 12 locally acquired cases in Florida [6]. The case numbers decreased in 2015, resulting in a total of 896 cases of CHIKV-induced disease and 1 locally acquired case in Texas [8]. This demonstrates a significant risk of outbreak in the US because of the ongoing epidemic in the Americas and the widespread presence of competent mosquito vectors [1].

While there have been millions of people affected by CHIKV within the last decade, methods of prevention and treatment are still lacking. Preventive measures focus on the mosquito vector and include recommendations such as wearing long sleeves, making use of mosquito nets, elimination of mosquito breeding sites and use of insecticides [9]. Several vaccines are in various stages of development, though none are yet commercially available [10 - 16]. Treatments are generally symptomatic and supportive, and the efficacies of potentially more specific therapies are currently unknown $[17,18]$.

\section{Disease Manifestations}

Following an incubation period of 2-7 days, approximately $95 \%$ of infected people will develop symptoms [9, 19]. These consist of high fever, headaches, rash, myalgia and severe joint pain, for which the virus was named; "chikungunya" is a Makonde word in Tanzania meaning "to walk bent over" or "that which bends up" [20]. In most cases these symptoms will resolve in approximately 2 weeks [9]. However, as many as $88 \%$ of people can have arthralgia persisting for 1 month after the initial symptoms [21]. Additionally, the current literature reports that CHIKV can result in a severe chronic arthralgia and/or arthritis that can last months to years following the initial infection [21, 22]. While there is an association between initial CHIKV infection and chronic disease, a causal relationship has not been established at this time. However, potential causes of chronic CHIKV-induced arthritis have been postulated, including viral persistence, induction of autoimmune disease, and exacerbation of pre-existing joint disease [4, 23].

Additionally, while less common, severe manifestations including gastrointestinal disease, neurologic complications including meningoencephalitis and seizures, cardiovascular disease, hemorrhagic manifestations, and death have been reported [9, 17, 24 - 26]. These severe manifestations are more frequent in children, the elderly, and people that have underlying comorbidities [17, 27]. Recently, maternal-fetal transmission has also been reported, resulting in some neonatal mortality $[19,25]$. Research has indicated that these neonatal infections occur as a result of direct contact with blood of mothers that are viremic at the time of parturition, rather than via placental transfer of virus [19, 28]. These neonatal cases occurred in approximately 3 out of 1,000 births during the La Reunion outbreak [17] and often resulted in severe manifestation in infants, including seizures, hemorrhagic disease, and abnormalities in cardiac function [19, $26]$.

\section{Risk Factors for Severe Disease}

Numerous risk factors for development of severe disease in adult patients have been reported including age and gender [9, 29]. Disease is often considered to be more severe in adults than in children, though adults younger than 35 years appear to be protected [29, 30]. However, it should be noted that some studies have found no correlation between age and disease severity [9]. Similarly, while some studies have reported an increased risk of severe disease in women $[9,31]$, others have demonstrated no significant difference associated with gender [30, 32].

A relationship between some of the more unusual disease manifestations described above and presence of underlying comorbidities at the time of initial infection has been described in up to $71.6 \%$ of patients [32]. Some of the more commonly reported comorbidities include hypertension and respiratory or cardiovascular disease [9, 29]. Ischemic heart disease and diabetes have been implicated as risk factors for persistent joint disease [30, 32]. Pre-existing joint disease has also often been associated with an increased susceptibility to development of chronic CHIKV-associated arthritis [24]. In one multivariate model, the 3 variables that were independently associated with chronic CHIKVassociated joint disease were being 45 years old or older, the initial severity of joint pain, and underlying osteoarthritis [29]. However, in a cohort of patients that developed chronic arthralgia following the La Reunion outbreak, only $2.8 \%$ had pre-existing joint pain, indicating that there are likely other, as yet unidentified factors involved in the development of this manifestation [30]. An additional confounding factor is that presence or absence of pre-existing joint disease is often ascertained via self-reporting questionnaires, while thorough orthopedic evaluations at the time of or prior to 
initial infection are rarely performed [29, 33 - 36]. Therefore, an association between pre-existing joint disease and the risk of development of prolonged chronic CHIKV-associated disease is still unproven.

In addition to the existence of underlying joint disease at the time of infection, the severity of acute disease has also been linked to risk of development of persistent arthralgia and/or arthritis. The parameters of initial disease severity that have been correlated with chronic disease include presence of joint swelling, severity of joint pain, and a stronger Th1 response with concurrent weak Th2 response during the acute phase of disease [22, 29]. Other measures of initial disease severity during acute disease were not associated with development of chronic disease, including initial viral load, number of joints affected, and duration of hospitalization [30].

\section{JOINT DISEASE}

\section{Clinical Presentation}

\section{Symptoms}

One of the most consistent and debilitating manifestations of CHIKV-induced disease in people consists of an often widespread, severe and incapacitating arthralgia, occurring in $85-100 \%$ of people with symptomatic infection [25, 29, 30, 37]. In fact, this disease manifestation is so common in CHIKV-induced disease, it has been reported that the presence of concurrent high fever and arthralgia has a specificity of $99.6 \%$ and positive predictive value of $84.6 \%$ for the diagnosis of CHIKV infection [38]. However, it should be noted that other arboviruses such as dengue, Zika, and other alphaviruses can have similar clinical presentations, including fever and arthralgia. Additionally, some of these such as dengue, Zika, and CHIKV are currently co-circulating in the western hemisphere [39], thus care should be taken to definitively identify the cause in any case with this presentation.

In most cases of CHIKV infection, patients develop symmetric oligo or polyarthralgia within minutes to days following the initial onset of fever $[9,18,40]$. While any joint can be affected, those that are most commonly reported are the distal extremities such as wrists, metacarpal and interphalangeal joints, as well as the ankles and metatarsophalangeal joints $[18,20,36,40,41]$, though some studies have indicated that the knee is also commonly affected [9, 31, 36, 42]. While the majority of cases have this distribution, it is important to recognize that patient presentation can vary and include asymmetric polyarthritis, monoarthralgia, and involvement of less commonly affected joints, such as the elbow, hip, and shoulder, as well as vertebral pain in sacroiliac, lumbosacral, and cervical regions [9, 43, 44]. Arthralgia associated with CHIKV infection is often considered to be the result of tenosynovitis and enthesopathy [9, 37, 41] and can be associated with paresthesia of the overlying skin [24]. In many cases, these manifestations of arthralgia will resolve within 1 to 4 weeks after the initial onset $[20,22,25]$.

However, in a wide range of cases (1.6-89.7\%), patients can have chronic ongoing or relapsing joint disease lasting months to years after the initial disease onset $[21,22,27,29,30,32,35,37,40,42]$. The distribution of joint manifestations in the chronic form is similar to the acute, including symmetrical involvement of joints of the distal extremities, knee, shoulders, and rarely the back [20, 29, 32, 35, 37]. Again, these symptoms are often associated with the presence of synovitis and/or tenosynovitis, articular destruction has been rarely reported, and as many as $15 \%$ of patients develop Raynaud's syndrome [26, 27, 37, 41].

The mechanisms involved in the development of the chronic or relapsing form of CHIKV-associated joint disease are poorly understood. Incidences of chronic CHIKV-associated joint disease have been fairly commonly reported since the La Reunion outbreak, though in most reports concurrent underlying joint abnormalities present prior to or at the time of infection cannot be ruled out [27, 29, 30, 35]. Additionally, it is possible that some patients develop joint disease such as osteoarthritis or rheumatoid arthritis, completely unrelated to the previous CHIKV infection. Ambiguities in the pathogenesis of CHIKV-associated joint disease and the potential continued or permanent damage that may occur during acute infection make proper classification of chronic CHIKV-associated joint disease difficult. The potential mechanisms associated with chronic CHIKV-associated joint disease are unknown, and no animal model currently replicates this aspect of disease. Therefore, the association between CHIKV infection and chronic joint pain is still unclear.

\section{Diagnosis of CHIKV Infection}

As discussed, the clinical presentation of CHIKV-associated arthralgia during the acute stages can be quite similar to other arthritogenic alphaviruses and other tropical arboviruses such as dengue and Zika [39, 45]. Therefore, definitive 
diagnosis is required. Serological assays for detection of anti-CHIKV IgM or IgG can be performed during the first few weeks or months, respectively, though false negative results can occur depending on the stage of disease [46]. Because of the high viremia associated with disease (see Blood and Clinical Chemistry Laboratory Results below), reversetranscriptase polymerase chain reaction (RT-PCR) or virus isolation utilizing cell culture or animal inoculation can be performed during the acute stage, but is only useful at 1 to 2 weeks post-infection $[10,46]$.

\section{Blood and Clinical Chemistry Laboratory Results}

In the acute stages of the disease, results of a complete blood cell count can be variable, though leukopenia, often as a result of lymphopenia, is one of the most common presentations [9, 19, 25]. This will often persist for the first week of infection, and is occasionally accompanied by thrombocytopenia and an increased hematocrit [9, 19]. In most instances, these parameters will return to normal in the second week of infection [25], though a persistent lymphopenia 36 months after the initial infection has been rarely reported [30]. Blood chemistry results are often normal throughout the disease course, though increased liver enzymes including ALT and AST have been reported [9, 25].

A high viremia is common in infected patients, frequently ranging from $10^{8}$ to $10^{12}$ viral RNA copies $/ \mathrm{mL}$, and lasting up to 12 days after the initial onset of clinical symptoms [17, 24, 25, 31]. In many cases, patients with higher viremias had more severe alterations in the above-mentioned CBC and chemistry results [31]. Persistent viremias are not apparent during the chronic stage of disease [30,41]. Infected patients will often seroconvert and have measurable IgG levels within the first week, sometimes as early as 2 days post infection [24], and lasting for years after the initial infection [41]. Additionally, many studies have demonstrated persistent IgM levels in patients lasting 18 months or longer [24, 30, 32, 36, 41]. Neutralizing IgM antibodies can also be found in the synovial fluid of patients 3 months after the initial infection [27]. These findings suggest that continued immune stimulation could play a role in some cases of prolonged CHIKV-associated arthritis.

In fact, several studies have suggested that chronic CHIKV-associated arthritis is similar to rheumatoid arthritis (RA) [9, 40], in part due to similarities in clinical presentation and laboratory results. Many patients meet the requirements established by the American College of Rheumatology to be diagnosed with RA [35, 40]. Studies have demonstrated positivity for rheumatoid factor (RF) in 13.6-57.1\% of patients with chronic CHIKV-associated arthritis [20,37, 40], though others reported no RF positivity in any patients [35].

Other similarities in laboratory results shared by CHIKV and RA include variably elevated erythrocyte sedimentation rates, elevated C-reactive protein, and presence of anti-citrullinated protein antibodies [9, 20, 31, 35, 38, $40,47]$. However, none of these are consistent findings in patients with acute or chronic CHIKV-associated arthralgia. Thus the association between CHIKV infection and development of autoimmune diseases such as RA unclear [23]. In at least some of these cases, it is possible the development of RA in patients is completely unrelated to previous CHIKV infection.

\section{Alterations in Cytokines Associated with CHIKV Infection}

During acute stages of disease (4-7 dpi), numerous cytokines are reportedly elevated including IFN- $\alpha$, IL-1Ra, IL-2R, IL-6, IL-7, IL-8, IL-12, IL-15, IL-4, MIG/CXCL-9, MIP-1 $\alpha / C C L-3$, HGF, bFGF, G-CSF, GM-CSF, and eotaxin/CCL-11 [22, 31, 48]. Some of these cytokines, such as IL-1 $\beta$, IL-6, and RANTES have been correlated with disease severity $[31,49]$, while increased levels of others are correlated specifically with higher viral load, such as IFN$\alpha$, IL-6, IL-16, IL-17, IL-1Ra, MCP-1, IL-12, IP-10/CXCL-10, IL-18, IL-18bp [22, 31]. At later timepoints in acute disease (10-15 dpi), a shift towards Th2 cytokines such as IL-4 and eotaxin has been reported [31], though elevations in Th1 cytokines such as IL-12 can also occur [22]. Unfortunately, most of these studies do not specifically correlate cytokine profiles with joint manifestations, though Rulli et al. reported that patients experiencing polyarthralgia had higher levels of MCP-1, TNF- $\alpha$ and IFN- $\gamma$ [50].

During later stages of disease, elevations in some cytokines have been associated with ongoing disease and persistent arthralgia, including IL-1Ra, IL-17, IL-6, MCP-1, MIP-1 $\alpha$, MIP-1 $\beta$, IL-8, GM-CSF, IFN- $\alpha$, and IL-12 [22, $31,49,51,52]$. Increased levels of CXCL10 and CXCL9 have been associated with more severe disease at 6 months post-infection [53]. Interestingly, it has also been reported that patients who recovered from disease had higher levels of HGF and eotaxin during the recovery period than patients with continuing disease manifestations [22]. While the majority of these studies focused on serological analyses, IFN- $\alpha$, IL-6, MCP-1, IL-8, and MMP2 have been demonstrated within the synovium of a patient with chronic arthralgia, while not present in two fully recovered patients [22]. Unfortunately, many of the reports on cytokine profiles of patients yield differing results [22, 48, 51], so the 
significances of these findings and their roles in disease pathogenesis remain unclear. These disparities may reflect differences in post-infection timepoints and/or different techniques for analyzing cytokine levels.

\section{Clinical Evaluation via Advanced Imaging}

Much of the current literature focuses on the use of standard radiography for evaluation of joints in CHIKV-infected patients and results are often variable [9, 24, 35, 40, 41]. Some significant bony changes, such as obvious erosions, can be seen by 10-18 months post infection (p.i.), involving the subchondral bone of the hands and wrists, including the interphalangeal joints $[35,41]$; though many patients have completely normal radiographs during this same time period [40]. In some cases, patients who had normal radiographs at 10 months p.i. showed evidence of joint space narrowing and bone erosions years after the initial infection [40], indicating that early changes as a result of CHIKV-associated joint disease may not be detectable by radiography. In this study, $42.9 \%$ of patients had normal radiographs at the time of diagnosis of chronic CHIKV arthritis, while $57.1 \%$ had evidence of joint space narrowing and $23.8 \%$ had visible erosions [40]. However, at the follow-up 24 months later, only $19 \%$ of patients had normal radiographs, and the percentages of patients demonstrating joint space narrowing and erosions had both increased to $81 \%$ [40].

Additionally, in some individuals with normal radiographs, MRI identified bone erosions in the hands of 5 of 6 patients at the 24 month post-diagnosis timepoint [40], indicating MRI may be a more sensitive technique for early identification of joint disease. In fact, CHIKV-induced arthritis has been compared to RA in regard to some of the pathologic changes, and studies have indicated that radiographs of RA patients are often normal for 6-12 months after the initial symptoms arise, while erosions can be demonstrated by MRI less than 6 months after symptom onset [54].

MRI has also demonstrated significant bilateral periosteal inflammation, carpal edema, and synovitis, which might not have been apparent on radiographs [41]. Other lesions described on MRI of patients with CHIKV include joint effusion, tendinitis/tenosynovitis, bone marrow edema, and bone erosions affecting various regions including hands, wrists, knees, and shoulders [9, 35, 41]. Additionally, while radiography has been shown to be useful predominantly at the later timepoints as discussed above, in a report utilizing MRI, bone erosions, tenosynovitis, and joint effusions have been demonstrated within the first 1 to 2 months p.i., further supporting the utility of this modality at earlier timepoints $[2,27]$. In one study, $80 \%$ of patients demonstrated significant lesions associated with CHIKV-associated chronic arthritis by 10 months post-infection [35] and in severe cases, tearing of menisci and cruciate ligaments have also been described [35].

Ultrasonography has shown some utility for diagnosis of soft tissue disease associated with CHIKV-induced arthralgia. Chopra et al. described substantial tenosynovitis and enthesopathy in patients suffering from recurrent musculoskeletal discomfort in the months following CHIKV infection [20] and Mathew et al. have similarly described tenosynovitis and bursitis [36].

\section{CHIKV Replication and Cell Tropisms}

CHIKV enters the host cell by clathrin-dependent endocytosis, followed by release of nucleocapsid into the host cell cytoplasm after acidification of the endosome $[12,55]$. Much of what is known regarding CHIKV replication and virion assembly is extrapolated from experiments examining these aspects in other alphaviruses [56]. Replication of virus occurs within the cytoplasm, associated with cytopathic vacuoles formed from rearrangement of host membranes [57]. Virion assembly is not well characterized in CHIKV, but also occurs within the cytoplasm, followed by acquisition of a lipid bilayer envelope by budding through the host cell membrane $[55,56]$.

Data regarding natural human infections and viral tropisms and persistence is limited, though viral persistence in tissues is considered to be a potential mechanism of chronic disease. In acute stages, CHIKV antigen has been demonstrated within skeletal muscle progenitor cells of infected patients and is associated with muscle necrosis and inflammation [58]. During the acute phase, CHIKV can also be detected in circulating monocytes [59]. Additionally, persistent CHIKV infection in muscle satellite cells has been demonstrated 3 months after the resolution of the acute phase of disease [36]. Persistent infection has also been demonstrated in synovial macrophages as long as 18 months after the initial infection [60]. Other articles have reported the presence of CHIKV antigen and/or virus within fibroblasts of the joint capsule, skin, muscle fascia, and dermis, though the timeline in terms of infection in these is unclear [17]. 


\section{Histopathology/Pathology}

Little histopathology has been performed on joint samples from CHIKV-infected human patients. Those that have been reported describe vascular proliferation, perivascular macrophages, and synovial hyperplasia [37]. Macrophages also can extend into surrounding soft tissues, including connective and peritendinous tissues and muscle [22]. In acute stages of disease, CD4+ T helper cells are essential in the inflammatory process [60], and intense activation of these cells, in addition to dendritic cells (DC), natural killer cells (NK), and CD8+ T cells, have been associated with development of chronic arthralgia [58]. While synovitis is often assumed to be a major component of disease in chronic CHIKV-associated arthritis, Jaffar-Bandjee et al. reported that tenosynovitis, enthesopathy and periosteal inflammation are more common [24]. However, due to the paucity of histopathological data associated with human disease, it is difficult to draw conclusions regarding pathogenesis of disease manifestations.

\section{Treatment and Vaccines}

Currently, specific treatment modalities for acute and chronic CHIKV-induced disease do not exist, and the majority of treatment plans consist of supportive and symptomatic care. In acute stages of the disease, this consists predominantly of rest, antipyretics and analgesics [9]. Unfortunately, efficacy data for most treatment regimens are lacking [26]. Most rely on non-steroidal anti-inflammatory drugs (NSAIDs), whose efficacy can be quite variable, and resolution of acute arthralgia can occur with or without their use [27, 38]. Similarly, NSAIDs can have variable effects in chronic cases. In one study in Bangladesh, 2 of 6 patients treated acutely with NSAIDs had persistent pain at 2-3 months [38]. In some cases joint pain may resolve, but stiffness remains [3], perhaps suggesting permanent alterations in articular or peri-articular tissues resulting in decreased mobility. It should also be noted that aspirin is not recommended due to bleeding risk [12].

In cases refractory to NSAID treatment, short-term courses of corticosteroids have been attempted with variable success. While corticosteroid treatments are usually not recommended due to the potential for a severe rebound of arthritis and tenosynovitis after therapy is ended, in severe cases of inflammatory polyarthritis refractory to NSAID treatment, the benefits may outweigh the risks [61]. In some cases, steroids helped resolve symptoms of arthralgia, tenosynovitis, entrapment syndrome and Raynaud's syndrome [37], while in other cases, they had no effect [18].

Because of the similarities between chronic CHIKV-associated arthralgia and RA, some disease modifying antirheumatic drugs (DMARDs) such as methotrexate, sulfasalazine, leflunomide and hydroxychloroquine have been utilized [40]. Again, the efficacies of these drugs are unclear [18]. In some patients, 6 month treatments with methotrexate and hydroxychloroquine appeared to be of benefit [36]. In some cases, MRI demonstrated clear improvement in severity of joint swelling, and pain and tendon involvement continuing for 15 months after initiation [41]. Other cases have demonstrated almost complete resolution of symptoms in 4-6 months using methotrexate, sulfasalazine and hydroxychloroquine [37]. However, Bouquillard et al. reported that all patients in their cohort treated with DMARDs had demonstrated progression of disease evidenced by more severe radiographic lesions at follow-up [40].

A randomized trial utilizing chloroquine on Reunion Island demonstrated no difference in duration of febrile arthralgia or severity of viremia [19]. Interestingly, in this study, patients receiving chloroquine actually complained more about their arthralgia than the placebo group. Additionally, a randomized trial in India comparing the efficacy of chloroquine compared to meloxicam demonstrated no significant difference in pain reported in either group over the 24 week study period [62]. While patients in this study improved overall, the authors concluded that there was no advantage to use of hydroxychloroquine as compared to NSAIDs or steroids. Waymouth et al. reported that while chloroquine may be helpful in more chronic stages, when given during the acute phase there was no benefit and patients were more likely to report joint symptoms at 200 dpi [37]. Therefore, the efficacy and utility of chloroquine in cases of $\mathrm{CHIKV}$-induced disease remain unclear.

Neutralizing antibodies have also been attempted as treatment modalities in mouse models. If received prior to infection, viremia and virus levels in ankles were decreased to below the level of detection, however, when administered after infection, though viremia decreased, there were no effects on the levels of viral RNA in the inoculated foot [4]. While the utility of passive immunization using monoclonal and polyclonal neutralizing antibodies in vitro and in mouse models have demonstrated prophylactic protection from CHIKV infection, the utility in human patients either as a prophylaxis or as a treatment during acute disease is unknown [12, 63]. Similarly, while IFN- $\alpha$ treatment has been shown to be able to prevent development of CHIKV-associated arthritis in a mouse model, this 
efficacy only occurs when it is administered prior to infection [12].

The efficacies of specific antiviral medications are also unknown, though in vitro studies have demonstrated potential utility of ribavirin against alphaviruses [10]. In a small cohort of ten patients, decreased pain and swelling were reported in most patients [64]. However, larger scale, controlled efficacy studies have not been performed to assess the true clinical utility of ribavirin in treating CHIKV-induced disease.

Numerous vaccines have been developed, though most have only been tested in animals and none are commercially available [12]. A wide variety of vaccine platform strategies have been utilized including live attenuated virus, inactivated virus, chimeric virus, DNA, and virus-like particles (VLPs) [11, 12, 65]. While many have shown promise in animal models as demonstrated by production of neutralizing antibodies and protection against viral challenge, few have moved into clinical trials. In the past, some vaccines have been tested in humans, and while antibody responses had shown promise, these were not without adverse reactions [12]. More recently, two vaccines have entered the clinical trial stage, and both are VLP-based [65]. The VLPs for one of these are produced in HEK293T cells and results of a phase I dose escalation trial have been reported, demonstrating significant production of neutralizing antibodies and no severe adverse responses $[65,66]$. The other is a measles virus-vectored VLP vaccine that also demonstrated a good neutralizing antibody response and only mild to moderate adverse events in patients [65, 67]. While both of these vaccines show promise, more trials are necessary before the overall safety and efficacies can be determined.

\section{DISCUSSION}

Extensive work focusing on the acute stages of CHIKV infection and utilizing animal models has enhanced our current understanding of these early timepoints of disease. While our knowledge in this area has greatly increased in recent years, the role of $\mathrm{CHIKV}$ in development of more chronic stages of disease has yet to be elucidated. Additionally, pre-existing joint disease present at the time of initial infection and development of joint disease such as OA or RA unrelated to CHIKV infection cannot be ruled out. Viral persistence has also been suggested as a mechanism of chronic CHIKV joint disease. In support of the former, virus has been demonstrated within muscle satellite cells 3 months after resolution of the acute phase of disease and in synovial tissues 18 months after initial infection [10, 36]. Alternatively, induction of autoimmune disease following CHIKV infection has been put forth as a possible mechanism of chronic joint disease [23]. This hypothesis has been mainly based on the fact that some patients have been diagnosed with RA in the months to years following the initial infection [33,40]. While viral infections have been linked to development of RA and microbial infections are associated with development of autoimmune reactive arthritis and ankylosing spondylitis [9, 68], no link between CHIKV infection and induction of autoimmune disease has yet been demonstrated.

More research is necessary, including prospective analysis of acute and chronic clinical cases to help determine the potential role of previous CHIKV infection in chronic disease. It is essential that thorough clinical workups of patients during acute stages be performed to evaluate for existing disease. A thorough evaluation for any pre-existing joint disease at the time of initial presentation with CHIKV fever, coupled with multiple follow-up examinations at later timepoints of disease would greatly add to our understanding of CHIKV-induced disease. Because of the great variability in the clinical workups available in the literature, it is likely that patients that are currently classified as having chronic CHIKV-associated arthritis actually represent a heterogeneous group of conditions. In fact, a retrospective study examining patient outcome after the Reunion Outbreak demonstrated that patients with chronic postCHIKV joint disease could be variably diagnosed with RA, spondyloarthropathies, undifferentiated polyarthritis or other musculoskeletal disorders [34].

While more invasive methods such as synovial fluid samples and synovial biopsies could undoubtedly add significant information to the growing body of knowledge on CHIKV pathogenesis, these procedures are not without risk to the patients and may therefore require more substantial justification to be performed in prospective studies. Additionally, these techniques can cause some level of discomfort and anxiety for patients, thus obtaining volunteers for initial procedures and ensuring their participation at additional follow-up timepoints can be difficult. Because MRI is a non-invasive procedure and minimal discomfort for the patient is expected, volunteer participation in clinical studies utilizing this modality would likely be higher.

By utilizing advanced imaging techniques, particularly MRI, it will be possible to perform a more thorough evaluation of potentially affected joints and associated soft tissues in CHIKV patients with acute disease. Assessment of the extent of the acute lesions such as joint effusion, synovitis, tendinitis, enthesopathy or bone marrow edema may 
make it possible to identify people with more severe disease, potentially aiding in the choice of treatment strategies for those at greater risk for developing chronic or relapsing disease. Additionally, the comparison of these early MRI analyses with later evaluations in patients who subsequently develop chronic disease may help to further characterize disease pathogenesis.

However, the value of the more traditional and invasive methods of sampling, including synovial fluid collection and synovial biopsy should still be recognized. Because the acute presentation of CHIKV-associated disease as visualized by MRI is currently unknown, it is possible that scans performed at early timepoints will provide only minimal information in regard to disease status, or that the acute presentation will be variable among patients and therefore the interpretation of results challenging. Pairing of MRI scans results and synovial fluid samples or biopsies obtained during the acute phase would be ideal. Should this prove to be too difficult or impossible for clinical studies, these early scans may still provide valuable information, and can certainly be extremely useful to rule out pre-existing joint disease and when paired with follow-up scans during the chronic phase.

These initial assessments might also be useful for guiding treatment options in patients. In many instances of less severe manifestations of joint disease associated with CHIKV infection, symptomatic treatments with antipyretics, NSAIDs, analgesics, and/or corticosteroids have been shown to be effective in alleviating symptoms [3, 27, 37]. Visualization of more severe joint manifestations of disease via MRI analysis may help identify patients that would benefit from more aggressive therapies and/or be candidates for more frequent clinical follow-up evaluations.

\section{CONCLUSION}

Though it has become commonplace to state that CHIKV can result in chronic arthralgia and/or arthritis, the evidence for this is lacking and potential pathogeneses are unknown. It seems clear that joint pain could persist for weeks to a couple of months in some cases, but care should be taken to assume that there is a connection between initial CHIKV infection and chronic joint pain lasting months to years. Currently, in all available reports, pre-existing joint disease or development of an unrelated joint disease such as OA or RA cannot be ruled out. The use of advanced imaging techniques, in conjunction with currently accepted methods of detection and evaluation of CHIKV-infected patients, could fill a significant gap in regard to early identification of patients at risk for developing ongoing joint disease associated with CHIKV infection, and aid in the characterization of the pathogenesis of arthralgia/arthritis associated with infection.

There is currently no definitive evidence that treatments beyond conservative NSAID therapy in more severe cases have any efficacy. In cases refractory to pain alleviation by NSAIDs, weak opioids such as tramadol or codeine can be added [61]. While investigations into alternative treatments such as inhibitors of viral replication, anti-cytokine therapies, and siRNA have occurred, their efficacies and utilities in a clinical setting are still unknown $[12,52]$.

While animal models have been useful to study acute joint disease demonstrating evidence for viral persistence, no models exist for development of chronic joint disease associated with CHIKV infection. Therefore, to further elucidate the potential role of CHIKV in chronic cases and to establish the efficacy of alternative therapies, clinical studies are required. There is a great need for reporting clinical information associated with both acute and chronic cases.

\section{CONFLICT OF INTEREST}

The authors confirm that this article content has no conflict of interest.

\section{ACKNOWLEDGEMENTS}

Declared none.

\section{REFERENCES}

[1] Madariaga M, Ticona E, Resurrecion C. Chikungunya: bending over the Americas and the rest of the world. Braz Soc Infect Dis 2016; 20(1): 91-8. [http://dx.doi.org/10.1016/j.bjid.2015.10.004]

[2] Simon F, Parola P, Grandadam M, et al. Chikungunya infection: an emerging rheumatism among travelers returned from Indian Ocean islands. Report of 47 cases. Medicine (Baltimore) 2007; 86(3): 123-37. [http://dx.doi.org/10.1097/MD/0b013e31806010a5] [PMID: 17505252]

[3] Volpe A, Caramaschi P, Angheben A, et al. Chikungunya outbreakremember the arthropathy. Rheumatology (Oxford) 2006; 45(11): 1449-50. [http://dx.doi.org/10.1093/rheumatology/kel275] [PMID: 16920753] 
[4] Hawman DW, Stoermer KA, Montgomery SA, et al. Chronic joint disease caused by persistent Chikungunya virus infection is controlled by the adaptive immune response. J Virol 2013; 87(24): 13878-88. [http://dx.doi.org/10.1128/JVI.02666-13] [PMID: 24131709]

[5] W.H.O. Neglected Tropical Diseases. 2016. Available from: http://www.who.int/ neglected_diseases/diseases/en/ [Accessed on: March 20];

[6] CDC. Chikungunya Virus, 2014 final data for the United States. 2016. Available from: http://www.cdc.gov/chikungunya/geo/unitedstates-2014.html. [Accessed on: March 20];

[7] PAHO. Chikungunya: Statistic Data. 2016. Available from: http://www.paho.org/hq/index.php?option=com_topics\&view=readall\& cid=5927\&Itemid=40931\&lang=en. [Accessed on: April 15];

[8] CDC. Chikungunya Virus, 2015 final data for the United States. 2016. Available from: https://www.cdc.gov/chikungunya/geo/unitedstates-2015.html. [Accessed on: July 20];

[9] Ali Ou Alla S, Combe B. Arthritis after infection with Chikungunya virus. Best Pract Res Clin Rheumatol 2011; 25(3): 337-46. [http://dx.doi.org/10.1016/j.berh.2011.03.005] [PMID: 22100284]

[10] Burt FJ, Rolph MS, Rulli NE, Mahalingam S, Heise MT. Chikungunya: a re-emerging virus. Lancet 2012; 379(9816): 662-71. [http://dx.doi.org/10.1016/S0140-6736(11)60281-X] [PMID: 22100854]

[11] Ahola T, Couderc T, Ng LF, et al. Therapeutics and vaccines against chikungunya virus. Vector Borne Zoonotic Dis 2015; 15(4): 250-7. [http://dx.doi.org/10.1089/vbz.2014.1681] [PMID: 25897811]

[12] Thiberville SD, Moyen N, Dupuis-Maguiraga L, et al. Chikungunya fever: epidemiology, clinical syndrome, pathogenesis and therapy. Antiviral Res 2013; 99(3): 345-70

[http://dx.doi.org/10.1016/j.antiviral.2013.06.009] [PMID: 23811281]

[13] Cavrini F, Gaibani P, Pierro AM, Rossini G, Landini MP, Sambri V. Chikungunya: an emerging and spreading arthropod-borne viral disease. J Infect Dev Ctries 2009; 3(10): 744-52. [PMID: 20009275]

[14] Akahata W, Yang ZY, Andersen H, et al. A virus-like particle vaccine for epidemic Chikungunya virus protects nonhuman primates against infection. Nat Med 2010; 16(3): 334-8. [http://dx.doi.org/10.1038/nm.2105] [PMID: 20111039]

[15] Hallengärd D, Kakoulidou M, Lulla A, et al. Novel attenuated Chikungunya vaccine candidates elicit protective immunity in C57BL/6 mice. J Virol 2014; 88(5): 2858-66.

[http://dx.doi.org/10.1128/JVI.03453-13] [PMID: 24371047]

[16] Mallilankaraman K, Shedlock DJ, Bao H, et al. A DNA vaccine against chikungunya virus is protective in mice and induces neutralizing antibodies in mice and nonhuman primates. PLoS Negl Trop Dis 2011; 5(1): e928. [http://dx.doi.org/10.1371/journal.pntd.0000928] [PMID: 21264351]

[17] Labadie K, Larcher T, Joubert C, et al. Chikungunya disease in nonhuman primates involves long-term viral persistence in macrophages. J Clin Invest 2010; 120(3): 894-906. [http://dx.doi.org/10.1172/JCI40104] [PMID: 20179353]

[18] Foissac M, Javelle E, Ray S, Guérin B, Simon F. Post-Chikungunya rheumatoid arthritis, Saint Martin. Emerg Infect Dis 2015; $21(3)$ : 530-2. [http://dx.doi.org/10.3201/eid2103.141397] [PMID: 25695499]

[19] Sebastian MR, Lodha R, Kabra SK. Chikungunya infection in children. Indian J Pediatr 2009; 76(2): $185-9$. [http://dx.doi.org/10.1007/s12098-009-0049-6] [PMID: 19330307]

[20] Chopra A, Anuradha V, Lagoo-Joshi V, Kunjir V, Salvi S, Saluja M. Chikungunya virus aches and pains: an emerging challenge. Arthritis Rheum 2008; 58(9): 2921-2.

[http://dx.doi.org/10.1002/art.23753] [PMID: 18759351]

[21] Calabrese LH. Emerging viral infections and arthritis: the role of the rheumatologist. Nat Clin Pract Rheumatol 2008; 4(1): 2-3. [http://dx.doi.org/10.1038/ncprheum0679] [PMID: 18043599]

[22] Dupuis-Maguiraga L, Noret M, Brun S, Le Grand R, Gras G, Roques P. Chikungunya disease: infection-associated markers from the acute to the chronic phase of arbovirus-induced arthralgia. PLoS Negl Trop Dis 2012; 6(3): e1446. [http://dx.doi.org/10.1371/journal.pntd.0001446] [PMID: 22479654]

[23] Burt F, Chen W, Mahalingam S. Chikungunya virus and arthritic disease. Lancet Infect Dis 2014; 14(9): 789-90. [http://dx.doi.org/10.1016/S1473-3099(14)70869-2] [PMID: 25164188]

[24] Jaffar-Bandjee MC, Das T, Hoarau JJ, Krejbich Trotot P, Denizot M, Ribera A, et al. Chikungunya virus takes centre stage in virally induced arthritis: possible cellular and molecular mechanisms to pathogenesis. Microbes Infect/Inst Pasteur 2009; 11(14-15): 1206-8. [http://dx.doi.org/10.1016/j.micinf.2009.10.001]

[25] Higgs S, Ziegler SA. A nonhuman primate model of chikungunya disease. J Clin Invest 2010; 120(3): 657-60. [http://dx.doi.org/10.1172/JCI42392] [PMID: 20179348]

[26] Khasnis AA, Schoen RT, Calabrese LH. Emerging viral infections in rheumatic diseases. Semin Arthritis Rheum 2011; 41 (2): 236-46. [http://dx.doi.org/10.1016/j.semarthrit.2011.01.008] [PMID: 21440932] 
[27] Mizuno Y, Kato Y, Takeshita N, et al. Clinical and radiological features of imported chikungunya fever in Japan: a study of six cases at the National Center for Global Health and Medicine. J Infect Chemother 2011; 17(3): 419-23. [http://dx.doi.org/10.1007/s10156-010-0124-y] [PMID: 20862507]

[28] Couderc T, Chrétien F, Schilte C, et al. A mouse model for Chikungunya: young age and inefficient type-I interferon signaling are risk factors for severe disease. PLoS Pathog 2008; 4(2): e29. [http://dx.doi.org/10.1371/journal.ppat.0040029] [PMID: 18282093]

[29] Sissoko D, Malvy D, Ezzedine K, et al. Post-epidemic Chikungunya disease on Reunion Island: course of rheumatic manifestations and associated factors over a 15-month period. PLoS Negl Trop Dis 2009; 3(3): e389. [http://dx.doi.org/10.1371/journal.pntd.0000389] [PMID: 19274071]

[30] Schilte C, Staikowsky F, Couderc T, et al. Chikungunya virus-associated long-term arthralgia: a 36-month prospective longitudinal study. PLoS Negl Trop Dis 2013; 7(3): e2137. [http://dx.doi.org/10.1371/journal.pntd.0002137] [PMID: 23556021]

[31] Chow A, Her Z, Ong EK, et al. Persistent arthralgia induced by Chikungunya virus infection is associated with interleukin-6 and granulocyte macrophage colony-stimulating factor. J Infect Dis 2011; 203(2): 149-57. [http://dx.doi.org/10.1093/infdis/jiq042] [PMID: 21288813]

[32] Borgherini G, Poubeau P, Jossaume A, et al. Persistent arthralgia associated with chikungunya virus: a study of 88 adult patients on reunion island. Clin Infect Dis 2008; 47(4): 469-75. [http://dx.doi.org/10.1086/590003] [PMID: 18611153]

[33] Suhrbier A, Jaffar-Bandjee MC, Gasque P. Arthritogenic alphavirusesan overview. Nat Rev Rheumatol 2012; 8(7): 420-9. [http://dx.doi.org/10.1038/nrrheum.2012.64] [PMID: 22565316]

[34] Javelle E, Ribera A, Degasne I, Gaüzère BA, Marimoutou C, Simon F. Specific management of post-chikungunya rheumatic disorders: a retrospective study of 159 cases in Reunion Island from 20062012. PLoS Negl Trop Dis 2015; 9(3): e0003603. [http://dx.doi.org/10.1371/journal.pntd.0003603] [PMID: 25760632]

[35] Manimunda SP, Vijayachari P, Uppoor R, et al. Clinical progression of chikungunya fever during acute and chronic arthritic stages and the changes in joint morphology as revealed by imaging. Trans R Soc Trop Med Hyg 2010; 104(6): 392-9. [http://dx.doi.org/10.1016/j.trstmh.2010.01.011] [PMID: 20171708]

[36] Mathew AJ, Goyal V, George E, Thekkemuriyil DV, Jayakumar B, Chopra A. Rheumatic-musculoskeletal pain and disorders in a naïve group of individuals 15 months following a Chikungunya viral epidemic in south India: a population based observational study. Int J Clin Pract 2011; 65(12): 1306-12. [http://dx.doi.org/10.1111/j.1742-1241.2011.02792.x] [PMID: 22093538]

[37] Waymouth HE, Zoutman DE, Towheed TE. Chikungunya-related arthritis: case report and review of the literature. Semin Arthritis Rheum 2013; 43(2): 273-8.

[http://dx.doi.org/10.1016/j.semarthrit.2013.03.003] [PMID: 23608542]

[38] Hassan R, Rahman MM, Moniruzzaman M, et al. Chikungunya - an emerging infection in Bangladesh: a case series. J Med Case Reports 2014; 8: 67. [http://dx.doi.org/10.1186/1752-1947-8-67] [PMID: 24559283]

[39] Roth A, Mercier A, Lepers C, et al. Concurrent outbreaks of dengue, chikungunya and Zika virus infections - an unprecedented epidemic wave of mosquito-borne viruses in the Pacific 2012-2014. Euro Surveill 2014; 19(41) pii: 20929.

[40] Bouquillard E, Combe B. A report of 21 cases of rheumatoid arthritis following Chikungunya fever. A mean follow-up of two years. Joint Bone Spine 2009; 76(6): 654-7. [http://dx.doi.org/10.1016/j.jbspin.2009.08.005] [PMID: 19945329]

[41] Malvy D, Ezzedine K, Mamani-Matsuda M, et al. Destructive arthritis in a patient with chikungunya virus infection with persistent specific IgM antibodies. BMC Infect Dis 2009; 9: 200. [http://dx.doi.org/10.1186/1471-2334-9-200] [PMID: 20003320]

[42] Chopra A, Anuradha V, Ghorpade R, Saluja M. Acute Chikungunya and persistent musculoskeletal pain following the 2006 Indian epidemic: a 2-year prospective rural community study. Epidemiol Infect 2012; 140(5): 842-50. [http://dx.doi.org/10.1017/S0950268811001300] [PMID: 21767452]

[43] Kularatne SA, Weerasinghe SC, Gihan C, et al. Epidemiology, clinical manifestations, and long-term outcomes of a major outbreak of chikungunya in a hamlet in sri lanka, in 2007: a longitudinal cohort study. J Trop Med 2012. 2012: 639178.

[44] Lui NL, Leong HN, Thumboo J. Polyarthritis in four patients with chikungunya arthritis. Singapore Med J 2012; 53(4): 241-3. [PMID: 22511045]

[45] Villamil-Gomez WE, Gonzalez-Camargo O, Rodriguez-Ayubi J, Zapata-Serpa D, Rodriguez-Morales AJ. Dengue, chikungunya and Zika coinfection in a patient from Colombia. J Infect Public Health 2016; 9(5): 684-.

[46] Schwartz KL, Giga A, Boggild AK. Chikungunya fever in Canada: fever and polyarthritis in a returned traveller. CMAJ 2014; 186(10): 772-4. [http://dx.doi.org/10.1503/cmaj.130680] 
[47] Carmona RJ, Shaikh S, Khalidi NA. Chikungunya viral polyarthritis. J Rheumatol 2008; 35(5): 935-6. [PMID: 18412299]

[48] Wauquier N, Becquart P, Nkoghe D, Padilla C, Ndjoyi-Mbiguino A, Leroy EM. The acute phase of Chikungunya virus infection in humans is associated with strong innate immunity and T CD8 cell activation. J Infect Dis 2011; 204(1): 115-23. [http://dx.doi.org/10.1093/infdis/jiq006] [PMID: 21628665]

[49] Phuklia W, Kasisith J, Modhiran N, et al. Osteoclastogenesis induced by CHIKV-infected fibroblast-like synoviocytes: a possible interplay between synoviocytes and monocytes/macrophages in CHIKV-induced arthralgia/arthritis. Virus Res 2013; 177(2): 179-88. [http://dx.doi.org/10.1016/j.virusres.2013.08.011] [PMID: 24012515]

[50] Rulli NE, Rolph MS, Srikiatkhachorn A, Anantapreecha S, Guglielmotti A, Mahalingam S. Protection from arthritis and myositis in a mouse model of acute chikungunya virus disease by bindarit, an inhibitor of monocyte chemotactic protein-1 synthesis. J Infect Dis 2011; 204(7): 1026-30. [http://dx.doi.org/10.1093/infdis/jir470] [PMID: 21881117]

[51] Noret M, Herrero L, Rulli N, et al. Interleukin 6, RANKL, and osteoprotegerin expression by chikungunya virus-infected human osteoblasts. The J Infect Dis 2012; 206(3): 455-7.

[52] Chaaitanya IK, Muruganandam N, Sundaram SG, et al. Role of proinflammatory cytokines and chemokines in chronic arthropathy in CHIKV infection. Viral Immunol 2011; 24(4): 265-71. [http://dx.doi.org/10.1089/vim.2010.0123] [PMID: 21830898]

[53] Kelvin AA, Banner D, Silvi G, et al. Inflammatory cytokine expression is associated with chikungunya virus resolution and symptom severity. PLoS Negl Trop Dis 2011; 5(8): e1279. [http://dx.doi.org/10.1371/journal.pntd.0001279] [PMID: 21858242]

[54] McQueen FM. Magnetic resonance imaging in early inflammatory arthritis: what is its role? Rheumatology (Oxford) 2000; 39(7): 700-6. [http://dx.doi.org/10.1093/rheumatology/39.7.700] [PMID: 10908686]

[55] Singh SK, Unni SK. Chikungunya virus: host pathogen interaction. Rev Med Virol 2011; 21(2): 78-88. [http://dx.doi.org/10.1002/rmv.681] [PMID: 21412934]

[56] Solignat M, Gay B, Higgs S, Briant L, Devaux C. Replication cycle of chikungunya: a re-emerging arbovirus. Virology 2009 ; $393(2)$ : $183-97$. [http://dx.doi.org/10.1016/j.virol.2009.07.024] [PMID: 19732931]

[57] Rupp JC, Sokoloski KJ, Gebhart NN, Hardy RW. Alphavirus RNA synthesis and non-structural protein functions. J Gen Virol 2015; 96(9): 2483-500.

[http://dx.doi.org/10.1099/jgv.0.000249] [PMID: 26219641]

[58] Assuncao-Miranda I, Cruz-Oliveira C, Da Poian AT. Molecular mechanisms involved in the pathogenesis of alphavirus-induced arthritis. BioMed Res Int 2013. 2013:973516 [http://dx.doi.org/10.1155/2013/973516]

[59] Her Z, Malleret B, Chan M, Ong EK, et al. Active infection of human blood monocytes by Chikungunya virus triggers an innate immune response. J Immunol (Baltimore, Md : 1950) 2010; 184(10): 5903-13 . [http://dx.doi.org/10.4049/jimmunol.0904181]

[60] Chen W, Foo SS, Sims NA, Herrero LJ, Walsh NC, Mahalingam S. Arthritogenic alphaviruses: new insights into arthritis and bone pathology. Trends Microbiol 2015; 23(1): 35-43. [http://dx.doi.org/10.1016/j.tim.2014.09.005] [PMID: 25449049]

[61] Simon F, Javelle E, Cabie A, et al. French guidelines for the management of chikungunya (acute and persistent presentations). November 2014. Med Mal Infect 2015; 45(7): 243-63. [http://dx.doi.org/10.1016/j.medmal.2015.05.007] [PMID: 26119684]

[62] Chopra A, Saluja M, Venugopalan A. Effectiveness of chloroquine and inflammatory cytokine response in patients with early persistent musculoskeletal pain and arthritis following chikungunya virus infection. Arthritis Rheumatol 2014; 66(2): 319-26. [http://dx.doi.org/10.1002/art.38221] [PMID: 24504804]

[63] Couderc T, Lecuit M. Chikungunya virus pathogenesis: From bedside to bench. Antiviral Res 2015; 121: 120-31. [http://dx.doi.org/10.1016/j.antiviral.2015.07.002] [PMID: 26159730]

[64] Ravichandran R, Manian M. Ribavirin therapy for Chikungunya arthritis. J Infect Dev Ctries 2008; 2(2): 140-2. [http://dx.doi.org/10.3855/T2.2.140] [PMID: 19738340]

[65] Morrison CR, Plante KS, Heise MT. Chikungunya virus: current perspectives on a reemerging virus. Microbiol Spectr 2016; 4(3) [http://dx.doi.org/10.1128/microbiolspec.EI10-0017-2016] [PMID: 27337473]

[66] Chang LJ, Dowd KA, Mendoza FH, et al. Safety and tolerability of chikungunya virus-like particle vaccine in healthy adults: a phase 1 doseescalation trial. Lancet 2014; 384(9959): 2046-52. [http://dx.doi.org/10.1016/S0140-6736(14)61185-5] [PMID: 25132507]

[67] Ramsauer K, Schwameis M, Firbas C, et al. Immunogenicity, safety, and tolerability of a recombinant measles-virus-based chikungunya vaccine: a randomised, double-blind, placebo-controlled, active-comparator, first-in-man trial. Lancet Infect Dis 2015; 15(5): 519-27. [http://dx.doi.org/10.1016/S1473-3099(15)70043-5] [PMID: 25739878] 
[68] Ajene AN, Fischer Walker CL, Black RE. Enteric pathogens and reactive arthritis: a systematic review of Campylobacter, salmonella and Shigella-associated reactive arthritis. J Health Popul Nutr 2013; 31(3): 299-307. [http://dx.doi.org/10.3329/jhpn.v31i3.16515] [PMID: 24288942]

(C) Goupil and Mores; Licensee Bentham Open

This is an open access article licensed under the terms of the Creative Commons Attribution-Non-Commercial 4.0 International Public License (CC BY-NC 4.0) (https://creativecommons.org/licenses/by-nc/4.0/legalcode), which permits unrestricted, non-commercial use, distribution and reproduction in any medium, provided the work is properly cited. 